\title{
O uso de Realidade Aumentada aplicada às disciplinas de Ciências Exatas no ensino médio brasileiro: Um Mapeamento Sistemático da Literatura
}

\author{
Sara das Mercês Silva ${ }^{1}$, Geisiane S. Matos ${ }^{1}$, \\ Fabíola Pantoja O. Araujo ${ }^{1}$, Josivaldo S. Araujo ${ }^{1}$ \\ ${ }^{1}$ Instituto de Ciências Exatas e Naturais (ICEN) \\ Universidade Federal do Pará (UFPA) \\ Rua Augusto Correa, 01 - 66075-110 - Belém - PA - Brasil \\ \{maru.merces18, geisi.mattos.s\}@gmail.com, \{fpoliveira, josivaldo\}@ufpa.br
}

\begin{abstract}
Augmented Reality (AR) has already been used in the teaching. In order to analyze the research and methodologies that already exist, this work carried out a Systematic Mapping of Literature to investigate the use of AR in the teaching of Exact Sciences. The search in databases, in the years 2016 until July 2020, returned 74 articles, of which 16 were analyzed. The results showed that the majority of studies use AR in the teaching of Math and took place in the South of the country.
\end{abstract}

Resumo. A Realidade Aumentada (RA) já vem sendo utilizada no ensino básico. Para que se analise as pesquisas e metodologias que já existem, o presente trabalho realizou um Mapeamento Sistematico da Literatura para investigar $o$ uso da RA no ensino de Ciências Exatas. A busca nas bases de dados, nos anos de 2016 até julho de 2020, retornou 74 artigos, dos quais 16 foram analisados. Os resultados mostraram que a maioria dos estudos utilizam RA na Matemática e aconteceram no Sul do país.

\section{Introdução}

O uso de recursos da Computação no ensino básico brasileiro, vêm crescendo nos últimos anos, como ferramenta de ajuda no processo de ensino-aprendizagem de diferentes disciplinas [das Mercês Silva et al. 2019]. Entre as ferramentas e metodologias que podem ser aplicadas no ensino-aprendizagem, a Realidade Aumentada (RA) aparece como um meio de simplificar a interação entre o mundo real e o virtual segundo [França and da Silva 2017], pois potencializa objetos do mundo real melhorando a interação.

A RA pode ajudar no ensino de disciplinas que abragem conteúdos mais abstratos como, por exemplo, disciplinas de Ciências Exatas. [Palhano et al. 2019] fala que a RA é um instrumento didático que permite a compreensão de conceitos e contextos que comumente parecem abstratos. Em [Detroz et al. 2015] afirma-se que pesquisadores brasileiros vêm utilizando estudos secundários, como Mapeamentos Sistemáticos da Literatura (MSL), para analisar o cenário da pesquisa do uso da RA no ensino de Ciências da Natureza e Matemática. Em vista disso o objetivo deste artigo é realizar um Mapeamento Sistemático da Literatura quanto ao uso da Realidade Aumentada aplicada às disciplinas 
de Ciências Exatas no ensino médio brasileiro, visando mapear as metodologias utilizadas, se a interdisciplinaridade está presente, quais disciplinas de Ciências Exatas o uso de RA é mais comum, assim como quais ferramentas estão sendo desenvolvidas e em quais regiões brasileiras as pesquisas estão acontecendo.

\subsection{Trabalhos correlatos}

Há na literatura trabalhos secundários sobre RA, no entanto, não foram identificados relatos sobre o uso de RA no ensino de disciplinas de Ciências Exatas, incluindo Matemática, no ensino médio brasileiro. A respeito das ferramentas de Realidade $\mathrm{Au}$ mentada aplicadas à Educação e o impacto que isso causa no processo de ensino e aprendizagem,[Silva et al. 2019] produziram uma Revisão Sistemática da Literatura (RSL) (que diferencia-se da MSL pois não só identifica como avalia os estudos encontrados na literatura) sobre o impacto do uso da RA no processo de aprendizagem. Para isso realizou uma busca de artigos, publicados no período de 2009 - 2017. Os resultados mostraram um aumento de trabalhos que avaliam o impacto positivo causado na educação com o uso da RA.

Quanto a analisar como a RA vem sendo utilizada na Educação, incluindo ensino superior, [Lopes et al. 2019] realizaram uma RSL no período de 2002 - 2017. A RSL resultou em 44 artigos, as principais práticas de RA identificadas neste trabalho estão nas seguintes áreas: Ciências da Saúde, Engenharia, Arquitetura e Design, as maiores dificuldades desta técnica, está o fato dos professores não dominarem as ferramentas e os softwares. A respeito do uso de RA em Ciências,[Lima et al. 2017] realizaram uma RSL analisando-a como prática voltada para o Ensino Fundamental. Neste trabalho, foi feita uma busca no Portal de Periódicos CAPES, sendo selecionados 16 artigos no ano de 2016. Os resultados mostraram que o uso da Realidade Aumentada contribui para o aprendizado dos alunos em Ciências. Em [Queiroz et al. 2019] foi investigado, por meio de um MSL, como a RA auxilia no processo de ensino e aprendizagem de Ciências da Natureza. Para isso, analisaram estudos primários no período de 2009 - 2018. Os resultados indicaram que há poucos trabalhos aplicados às Ciências da Natureza, principalmente no CentroOeste brasileiro. Os estudos secundários relatados aqui, mostram que o uso da Realidade Aumentada vêm crescendo nos últimos anos, em diferentes contextos educacionais brasileiros, porém ainda existem carências em pesquisas, tanto em trabalhos primários quanto secundários. O presente trabalho realizou um Mapeamento Sistemático da Literatura para investigar o uso da RA no ensino de Ciências Exatas, incluindo Matemática, no contexto do Ensino Médio (EM). Para isso, foi realizada uma busca em diversas bases de dados no período compreendido entre 2016 e o primeiro semestre de 2020 , pois o objetivo foi analisar os estudos primários mais recentes. Os resultados encontrados são discutidos na Seção 3.

Este artigo está estruturado da seguinte maneira: Na Seção 2 é descrita a metodologia usada no MSL, na Seção 3 são expostos os resultados do Mapeamento. Por fim, na Seção 4 são apresentadas as considerações finais deste trabalho.

\section{Metodologia do Mapeamento Sistemático da Literatura (MSL)}

A metodologia aplicada para identificar estudos primários a respeito do uso da RA nas disciplinas de Ciências Exatas no EM, é respaldada no Mapeamento Sistemático da Li- 


\begin{tabular}{|c|}
\hline Questões Específicas (QE) \\
\hline QE1. Quais são os métodos de avaliação aplicados pelos autores? \\
\hline QE2. Quais as metodologias aplicadas? \\
\hline QE3. Quais são as instituições dos pesquisadores e como elas estão distribuídas no Brasil? \\
\hline QE4.Quais as ferramentas desenvolvidas e/ou utilizadas na pesquisa? \\
\hline QE5. Os alunos desenvolveram as atividades individualmente ou equipe? \\
\hline QE6. Quais disciplinas de Ciências, incluindo a Matemática, a RA foi utlizada? \\
\hline QE7. Quais dificuldades encontradas? E como elas foram identificadas? \\
\hline
\end{tabular}

Tabela 1. Questões Específicas

teratura (MSL). Os procedimentos metodológicos percorridos aqui, são referentes à metodologia apresentada na pesquisa de [Cabrejos et al. 2018]. Nas seções seguintes são discutidas as etapas de definição do Protocolo de Pesquisa e execução do Estudo Secundário.

\subsection{Definição do Protocolo de Pesquisa}

O Protocolo de Pesquisa é o que descreve todos os passos e métodos necessários para a construção do MSL. Nesta etapa, definiu-se o tema principal, as questões de pesquisa, bases de dados, strings de busca e critérios de inclusão e exclusão.

\subsubsection{Questões de Pesquisa}

O objetivo de um estudo secundário é identificar estudos primários, extrair e analisar dados destes estudos [Cabrejos et al. 2018]. Para isso, é muito importante a elaboração de uma Questão de Pesquisa (QP) objetiva e precisa o suficiente para ajudar no processo de pesquisa e extração de dados. A Questão de Pesquisa foi: QP1 "Quais metodologias e ferramentas vêm sendo utilizadas pelos professores no Ensino Médio brasileiro para aplicar a Realidade Aumentada (RA), de maneira interdisciplinar ou não, no ensino de Ciências Exatas, incluindo Matemática?'Para obter os resultados esperados, por meio do MSL, além da Questão de Pesquisa principal, elaborou-se Questões Específicas (QE), apresentadas na Tabela 1:

\subsubsection{Bases de Pesquisa e Strings de busca}

Como o foco do mapeamento é no uso da RA na Educação brasileira, as bases de pesquisa que estão disponíveis 4, são veículos de publicações brasileiras na área de Informática na Educação. A exceção são os anais do Congresso Internacional de Informática Educativa (Tise), por se tratar de um evento latino-americano que recebe um considerável quantitativo de trabalhos brasileiros. Optou-se por utilizar três strings de busca (uma mesclando termos em português e em inglês, a segunda apenas em português e a terceira apenas em inglês), pois muitos trabalhos brasileiros são publicados escritos em inglês e percebeu-se que cada uma das strings utilizadas, retornavam trabalhos distintos. As strings de busca foram aplicadas na pesquisa automática, assim como na busca manual, e são apresentadas a seguir, na 2. 


\begin{tabular}{|c|}
\hline Strings de busca \\
\hline $\begin{array}{c}\text { ((Realidade Aumentada OR Augmented Reality) AND (Educa* OR Ensino) AND (Ciência* OR } \\
\text { Matemática OR Química OR Física OR Science OR Math* OR Physics OR Chemistry)) }\end{array}$ \\
\hline ((Realidade Aumentada) AND (Educa* OR Ensino) AND (Ciência* \\
OR Matemática OR Química OR Física)) \\
\hline ((Augmented Reality) AND (Educa*) AND (Science OR Math* \\
OR Physics OR Chemistry))
\end{tabular}

Tabela 2. String de busca

\begin{tabular}{|c|c|}
\hline Critérios de Inclusão (CI) & Critérios de Exclusão (CE) \\
\hline CI1. É uma pesquisa nacional & CE1. Não é uma pesquisa nacional \\
\hline $\begin{array}{c}\text { CI2. É uma pesquisa de 2016 ou ano posterior } \\
\text { (até primeiro semestre de 2020) }\end{array}$ & CE2. É uma pesquisa anterior à 2016 \\
\hline $\begin{array}{c}\text { CI3. Aborda o uso de RA em Quím. } \\
\text { /Fís./Mat. }\end{array}$ & CE3 Não Aborda o uso de RA em Quím./ Fís./ Mat. \\
\hline CI4. É um artigo completo & CE4. Não é um artigo completo. \\
\hline $\begin{array}{c}\text { CI5. O público alvo são alunos/ prof. } \\
\text { do EM. }\end{array}$ & CE5. O público alvo não são alunos/ prof. do EM \\
\hline CI6. É um estudo primário & CE6. É um estudo secundário. \\
\hline
\end{tabular}

Tabela 3. Critérios de Inclusão e Exclusão

\subsubsection{Critérios de inclusão e exclusão}

Após as pesquisas automática (string de busca) e manual nas bases de pesquisa, os critérios de inclusão e exclusão são utilizados para fazer a filtragem nos estudos retornados. Nessa atividade, os autores da pesquisa aplicaram os critérios de inclusão e exclusão, individualmente. Os critérios aplicados, nesse MSL, estão na 2.

\subsection{Execução do Estudo Secundário}

Para planejar e executar o estudo secundário, utilizou-se o Parsifal ${ }^{1}$. Esta ferramenta ajuda no processo de Mapeamentos ou Revisões Sistemáticos da Literatura e sua escolha recai no fato dela ser gratuita, está disponível online e apresentar facilidade em compartilhar com todos os pesquisadores as informações do MSL. A aplicação das buscas automáticas e manuais nas bases de pesquisa, entre os anos de 2016 e primeiros semestre de 2020, retornou 74 artigos e um total de 16 artigos passaram nos critérios de inclusão e exclusão. O detalhamento desta etapa está disponível na 4 e 5. Todas as informações dos artigos filtrados e seus metadados estão organizados na ferramenta Parsifal.

\section{Resultados}

Nesta seção estão expostas as respostas para a Questão de Pesquisa (QP) principal e as sete Questões Específicas (QE). Essas respostas são resultado da extração de dados dos 16 estudos primários aceitos no Mapeamento Sistemático da Literatura, que estão disponíveis na 5 .

\footnotetext{
${ }^{1}$ https://parsif.al/
} 
IX Congresso Brasileiro de Informática na Educação (CBIE 2020)

Anais do XXVI Workshop de Informática na Escola (WIE 2020)

\begin{tabular}{|c|c|c|}
\hline Bases de Pesquisa & Artigos Retornados & Artigos Aceitos \\
\hline Revista Brasileira de Informática na Educação & 01 & 0 \\
\hline Revista de Informática Teórica e Aplicada & 03 & 01 \\
\hline Revista Informática na Educação: Teoria e Prática & 01 & 0 \\
\hline Revista Novas Tecnologias na Educação & 17 & 08 \\
\hline Revista principia - IFPB & 0 & 0 \\
\hline Revista de Educação, Ciência e Tecnologia & 14 & 01 \\
\hline Redin - Revista Educacional Interdisciplinar & 3 & 01 \\
\hline Revistas IFG & 01 & 01 \\
\hline Revistas PUC SP & 04 & 01 \\
\hline Simpósio Brasileiro de Informática na Educação & 15 & 01 \\
\hline Workshop Desafios da Comp. Aplicada à Educação & 0 & 0 \\
\hline Workshop de Informática na Escola & 06 & 0 \\
\hline Computer on the Beach & 03 & 0 \\
\hline Anais dos Workshops do CBIE & 05 & 01 \\
\hline XI Encontro Nacional Pesquisa Educação em Ciências & 01 & 01 \\
\hline
\end{tabular}

Tabela 4. Bases de Pesquisa e quantitativo de artigos retornados e aceitos (filtrados).

\subsection{QP1.'Quais metodologias e ferramentas vêm sendo utilizadas pelos professores no Ensino Médio brasileiro para aplicar a Realidade Aumentada (RA), de maneira interdisciplinar ou não, no ensino de Ciências Exatas, incluindo Matemática ?"}

Os resultados obtidos, por meio das informações extraídas dos estudos analisados, mostram que as metodologias utilizadas para aplicar a RA no ensino de Ciências Exatas são as seguintes: pesquisa bibliográfica, desenvolvimento de softwares de RA ou uso de softwares já prontos (a maioria jogos para celular) e aplicação desses softwares nas aulas de Física, Química e Matemática para ajudar na visualização de objetos em 3D. Foram utilizadas sequências didáticas em algumas pesquisas. No processo de avaliação, os estudos primários realizaram testes com usuários (alunos e/ou professores do ensino médio), observação das atividades e uso de questionários. Nota-se que poucos pesquisadores desenvolveram atividades interdisciplinares ou em grupo e que a maiorias das pesquisas são para o ensino de trigonometria em Matemática. Essas informações estão detalhadas a seguir, por meio das Questões de Pesquisas específicas.

\subsection{QE3.’Quais são as instituições envolvidas na área e como elas estão distribuídas no Brasil?"”}

As instituições dos estudos primários selecionandos são, em sua maioria, públicas federais e alguns estudos foram desenvolvidos por pesquisadores de instituições não-públicas mas que trabalharam juntos na mesma pesquisa. Foi identificado o quantitativo de 14 instituições, sendo 11 Universidades ou Institutos Federais (UFRGS, UFSC, UFBA, UFMA, UFPR, UFPB, UFSE IFSC, IFG UNIVASF e UEZO) e três instituições privadas (PUCRS, USS e Estácio de Sá). Os 16 estudos aceitos estão distribuídos regionalmente pelo Brasil da seguinte forma: Nordeste (quatro estudos), Sul (nove estudos), Sudeste (um estudo) e Centro-Oeste (um estudo). Duas das pesquisas são fruto de parcerias de 
IX Congresso Brasileiro de Informática na Educação (CBIE 2020)

Anais do XXVI Workshop de Informática na Escola (WIE 2020)

\begin{tabular}{|c|c|}
\hline Título Resumido & Referências \\
\hline 01 GeometriAR: aplicativo educacional com RA & [Gomes et al. 2019] \\
\hline 02 Utilização da RA em aulas de física & [Denardin and Manzano 2018] \\
\hline 03 Usando Smartphone e RA para Geometria espacial & [Macedo et al. 2017] \\
\hline 04 RA na Educação: Análise de Flaras e Aumentaty & [Júnior and Dantas 2018] \\
\hline 05 A integração da RA em sala de aula & [Macedo and Góes 2019] \\
\hline 06 Um Jogo Móvel Baseado em Localização & [Laurindo et al. 2020] \\
\hline 07 Estudo Exploratório no Ensino de Física & [Nicolete et al. 2019] \\
\hline 08 Mobile-learning: aprendizagem matemática & [Resende and Muller 2018] \\
\hline 09 Aplicações de RA no ensino de Física & [Denardin and Manzano 2016] \\
\hline 10 ALCity: Uma Ferramenta de Autoria & [Moura et al. 2017] \\
\hline 11 AR Lab: Augmented Reality App & [da Silva et al. 2019] \\
\hline 12 Contribuições da RA para o Ensino de Química & [de Almeida and da Silva 2017] \\
\hline 13 Análise das experiências educacionais dos estudantes & [Herpich and Tarouco 2019] \\
\hline 14 RA Como Apoio à Aprendizagem de Poliedros & [Silva and Vasconcelos 2019] \\
\hline 15 Avaliação do desenvol. de ambientes de RA & [Gonçalves et al. 2017] \\
\hline 16 Geotransform3d: Objeto Computacional EM RA & [Barbosa and de Alencar Carvalho 2017] \\
\hline
\end{tabular}

Tabela 5. Estudos primários analisados.

institutos privados e públicos: um estudo é resultado de uma parceria de pesquisadores do Instituto Federal de Santa Catarina (Sul) com Estácio de Sá - CEUT do Piauí (Nordeste) e o outro da parceria de pesquisadores da Universidade Severino Sombra (USS) e Centro Universitário Estadual da Zona Oeste (UEZO), ambos do Rio de Janeiro (Sudeste).

\subsection{QE6.’Quais disciplinas de Ciências Exatas, incluindo a Matemática, a RA foi utilizada ?"”}

Todas as pesquisas envolviam o uso de RA em disciplinas de Ciências Exatas, incluindo Matemática, no contexto do Ensino Médio. A maioria das pesquisas contemplava o ensino de Matemática (seis estudos), cinco estudos relatam o uso de RA no ensino de Física e três no ensino de Química. Um estudo apenas apresenta um software que pode ser usado por vários professores, em qualquer disciplina (incluindo Matemática, Física e Química), para criar atividades diversas e outro pode ser aplicado no ensino de Física e Matemática.

\subsection{QE2.'Quais as metodologias aplicadas?'}

As metodologias aplicadas nos 16 artigos estão retratadas a seguir. Vale ressaltar que os alunos desenvolveram as atividades individualmente em oito estudos e em equipe em quatro deles. Em um estudo, os professores apenas testaram a ferramenta e em outros três não há relatos de testes, se os alunos trabalharam individualmente ou não.

- Metodologia 01: Aplicou-se conceitos de Tecnologia na Educação e Realidade Aumentada, no desenvolvimento de um aplicativo para auxiliar nas aulas de laboratório de Química, foram realizados experimentos práticos com um grupo de alunos.

- Metodologia 02: Neste artigo construiu-se uma aplicação e avaliou-se a usabilidade da ferramenta. 
- Metodologia 03: Ministrou-se um minicurso para professores de Física, mostrando como aplicar a ferramenta na disciplina.

- Metodologia 04: Utilizando RA e o conteúdo matemático, desenvolveu-se um aplicativo para melhorar o estudo de mapas de contorno e geometria espacial.

- Metodologia 05: Investigou-se a contribuição do uso de Laboratórios Remotos Aumentados e Laboratórios Virtuais de RA para o ensino de circuitos, utilizando uma pesquisa exploratória de caráter explicativo.

- Metodologia 06: Levantamento de requisitos, implementação e testes da aplicação.

- Metodologia 07: Uma sequência de atividades, para analisar a integração da RA em dispositivos móveis no ensino e aprendizagem de Geometria espacial no Ensino médio.

- Metodologia 08: Foram elaboradas sequências didáticas de três disciplinas (História, Biologia e Química) utilizando a ferramenta Aumentaty.

- Metodologia 09: Construiu-se um aplicativo de RA para o estudo de pirâmides, uma sequência didática e um experimento envolvendo sua utilização em sala de aula.

- Metodologia 10: Utilizou-se a RA na disciplina de Física no terceiro ano do ensino médio. Algumas páginas do material didático da escola foram melhoradas com materiais de RA.

- Metodologia 11: A metodologia dividiu-se em duas partes. Na primeira utilizouse Engenharia de Software para desenvolver a aplicação, a segunda avaliou o aplicativo do ponto de vista de professores de Matemática.

- Metodologia 12: Usou a Teoria da Aprendizagem Significativa (TAS) para o ensino de Matemática através de uma aplicação de RA.

- Metodologia 13: Foram lecionadas aulas sobre o conteúdo de Física, em seguida os alunos foram divididos em grupos e aplicou-se as atividades utilizando RA.

- Metodologia 14: A metodologia aplicada foi de natureza qualitativa do tipo estudo de caso.

- Metodologia 15: A metodologia foi quasi-experimental e fez uso de uma aplicação já existente para o ensino de Física, onde os estudantes foram divididos em dois grupos: Grupo Controle (GC) e Grupo Experimental (GE).

- Artigo 16: Desenvolveu-se uma aplicação lúdica de RA para ajudar no ensino dos conteúdos que os alunos encontravam mais dificuldades.

\subsection{QE4.'Quais as ferramentas desenvolvidas e/ou utilizadas na pesquisa ?'}

Em algumas pesquisas foram desenvolvidas aplicações de RA para auxiliar no ensino das disciplinas de Física, Química e Matemática, em outras utilizaram ferramentas já disponíveis:

- Vuforia + Unity: Ferramentas para jogos aplicações em RA .

- Layar: Software para criação e editoração de conteúdos de RA (utilizado em dois dos 16 estudos analisados).

- C \#: Linguagem de programação..

- Aumentaty: utilizada para trabalhar empregando a RA.

- ARtoolkit: Ferramenta para desenvolver aplicações em RA.

Ferramentas desenvolvidas nas pesquisas 
- AR Lab: aplicativo de Realidade Aumentada para o ensino de Química

- ALCity: Uma Ferramenta de Autoria para o Desenvolvimento de Jogos Móveis.

- Mobile-learning: aprendizagem de matemática.

- O Laboratório Remoto Aumentada - Painel Elétrico.

- Um Jogo Móvel para Motivar e Acompanhar estudantes no EnsinoAprendizagem.

- Aplicativo PolyedRA para o ensino de Poliedros.

- AppiRAmide para o estudo de Geometria espacial por meio de RA.

- GeometriAR: aplicativo educacional com RA para auxiliar o ensino de sólidos geométricos.

- GeoTransform3D: aplicativo para o ensino de Matemática.

- Ava-tAR: aplicativo educacional de RA para o ensino de Física.

\subsection{QE1.'Quais são os métodos de avaliação aplicados pelos autores (se houver algum) e dificuldades encontradas ?"}

Os métodos de avaliação mais utilizados nos estudos (12 estudos) analisados foram experimentos práticos em sala de aula com alunos da disciplina, fazendo uso das ferramentas e metodologias elaboradas pelos pesquisadores. Em sete pesquisas os autores usaram o experimento prático junto com questionários e testes para avaliar se as ferramentas ajudaram na apredizagem, satisfação e possíveis dificuldades dos usuários . Os resultados dos testes mostraram aceitabilidade e satisfação dos usuários em relação as metodologias e ferramentas aplicadas. Dois estudos utilizaram, como método de avaliação, o desenvolvimento de atividade por professores, com o intuito de identificar se as ferramentas e metodologias desenvolvidas poderiam ser aplicadas aos alunos nas aulas. Um estudo realizou testes com especialistas na área, afim de avaliar a aplicação desenvolvida e um estudo não relatou métodos de avaliação. Quanto às dificuldades, quatro pesquisas não relataram dificuldades, apenas sugestões de melhorias para trabalhos futuros e as outras pesquisas relataram dificuldades relacionadas à falta de Internet e smartphone para todos os alunos, incapacidade das câmeras dos celulares realizarem a leitura de marcadores e problemas de usabilidade dos aplicativos, como a falta de informações para os usuários.

\section{Considerações Finais}

Esse artigo apresentou um Mapeamento Sistemático da Literatura do uso de Realidade Aumentada em disciplinas de Física, Química e Matemática, no Ensino Médio brasileiro. Após a aplicação do protocolo de MSL, 16 estudos primários foram analisados por meio de questões específicas. Os resultados mostraram que os estudos foram desenvolvidos principalmente nas regiões Nordeste e Sul do país, por instituições federais de ensino, sendo que nenhum trabalho ocorreu no Norte do Brasil, o que evidencia a carência de pesquisas utilizando RA no ensino de Ciências Exatas nessa região. Além disso, identificou-se que há necessidade de mais estudos voltados para a disciplina de Química e as principais dificuldades enfrentadas, por pesquisadores e o público alvo, foi a falta de acesso à Internet e celulares. As metodologias aplicadas nas pesquisas, em sua maioria, focaram em testes e experimentos práticos, apresentando aceitabilidade e satisfação por parte de professores e alunos. Como trabalhos futuros, indica-se investigar o uso da Realidade Aumentada no ensino de Ciências Exatas no Ensino Médio de outros países e comparar as metodologias e resultados alcançados, com as metodologias e resultados dos estudos primários brasileiro, por meio de uma Revisão Sistemática da Literatura (RSL). 
IX Congresso Brasileiro de Informática na Educação (CBIE 2020)

Anais do XXVI Workshop de Informática na Escola (WIE 2020)

\section{Referências}

Barbosa, J. W. S. and de Alencar Carvalho, C. V. (2017). Geotransform3d: Objeto computacional em realidade aumentada para apoio ao ensino da matemÁtica. Revista de Educação, Ciências e Matemática, pages 226-239.

Cabrejos, L. J. E. R., Viana, D., and dos Santos, R. P. (2018). Planejamento e execução de estudos secundários em informática na educação: Um guia prático baseado em experiências. Anais da Jornada de Atualização em Informática na Educação, pages $21-52$.

da Silva, B. R., Zuchi, J. H., Vicente, L. K., Rauta, L. R. P., Nunes, M. B., Pancracio, V. A. S., and Junior, W. B. (2019). Ar lab: Augmented reality app for chemistry education. Nuevas Ideas en Informática Educativa, pages 71-77.

das Mercês Silva, S., a Costa Araújo, F., Santos, S., Pires, Y., Seruffo, M., and Araujo, J. (2019). Desenvolvimento de jogos como mecanismo pedagógico: Um relato de experiência na escola pública visando à sustentabilidade e preservação do meio ambiente. pages 637-646.

de Almeida, S. H. and da Silva, C. C. (2017). Contribuições da realidade aumentada para o ensino de quÍmica no ensino mÉdio do ifg cÂmpus jatál. Anais da Semana de Licenciatura, pages 253-267.

Denardin, L. and Manzano, R. (2016). Aplicações de realidade aumentada no ensino de física a partir do software layar. RENOTE, pages 1-10.

Denardin, L. and Manzano, R. (2018). Desenvolvimento, utilização e avaliação da realidade aumentada em aulas de física. RENOTE, pages 1-10.

Detroz, J., Hinz, M., and Hounsell, M. (2015). Uso de pesquisa bibliográfica em informática na educacão: um mapeamento sistemático. Revista Brasileira de Informática na Educação, pages 28-38.

França, C. R. and da Silva, T. (2017). A utilização da Realidade Virtual e Aumentada no Ensino de Ciências no Brasil. Programa de Pós-Graduacao em Educação Científica e Tecnológica - Universidade Federal de Santa Catarina (UFSC), s.n edition.

Gomes, A., Ramos, R., Brito, L., Batista, M., and Leal, B. (2019). Geometriar: aplicativo educacional com realidade aumentada para auxiliar o ensino de sólidos geométricos. RENOTE, pages 405-414.

Gonçalves, R. L., de Oliveira, L. D., and Vettori, M. (2017). Avaliação do desenvolvimento de ambientes de realidade aumentada elaborados por alunos do ensino médio em aulas de física. XI Encontro Nacional de Pesquisa em Educação em Ciências - XI ENPEC, pages $1-8$.

Herpich, F. and Tarouco, L. (2019). Análise das experiências educacionais dos estudantes com simulações em realidade aumentada móvel para o desenvolvimento do conhecimento em física. pages 778-787.

Júnior, D. and Dantas, A. (2018). Realidade aumentada na educação: Uma análise das ferramentas flaras e aumentaty como recursos para aulas expositivas. RENOTE, pages 1-10. 
Laurindo, L., de Moura, I., and dos Santos, M. R. P. (2020). Um jogo móvel baseado em localização para motivar e acompanhar estudantes no processo de ensinoaprendizagem. pages 163-172.

Lima, M. B., Pereira, L. B., e Alves Silva, L., Meríno, C. G., and Struchiner, M. (2017). Realidade aumentada no ensino de ciências: uma revisão de literatura. XI Encontro Nacional de Pesquisa em Educação em Ciências - XI ENPEC, 11:1-11.

Lopes, L., Vidotto, K., Pozzebon, E., and Ferenhof, H. (2019). Inovações educacionais com o uso da realidade aumentada: Uma revisão sistemática. Educação em Revista, pages $1-33$.

Macedo, A. and Góes, A. (2019). A integração da realidade aumentada em sala de aula: a pesquisa aplicada em colégios públicos do litoral paranaense. RENOTE, pages 1-10.

Macedo, A., Silva, J., and Buriol, T. (2017). Usando smartphone e realidade aumentada para estudar geometria espacial. RENOTE, pages 1-10.

Moura, I., Nolêto, C., and Revoredo, I. (2017). Alcity: Uma ferramenta de autoria para o desenvolvimento de jogos móveis baseados em localização para apoiar o processo de ensino. pages 435-444.

Nicolete, P. C., Júnior, E., Cristiano, M., Tarouco, L., Vila, E., and Silva, J. (2019). Estudo exploratÓrio sobre realidade aumentada e laboratÓrio remoto no ensino de física. RENOTE, pages 345-355.

Palhano, M., de Oliveira, F., and Grossi, L. (2019). A realidade aumentada no ensino de sólidos geométricos. Anais do XXX Simpósio Brasileiro de Informática na Educação (SBIE 2019), pages 1012-1021.

Queiroz, E., Moura, R., and Souza, E. (2019). Como a realidade aumentada tem auxiliado no processo de ensino e aprendizagem de ciências da natureza? um mapeamento sistemático da literatura. In Anais do IV Congresso sobre Tecnologias na Educação, pages 1-10, Porto Alegre, RS, Brasil. SBC.

Resende, B. and Muller, T. (2018). Mobile-learning: aprendizagem matemática por meio de realidade aumentada. Revista de Educação Ciência e Tecnologia, pages 1-14.

Silva, M., Teixeira, J., Cavalcante, P., and Teichrieb, V. (2019). Perspectives on how to evaluate augmented reality technology tools for education: a systematic review. Journal of the Brazilian Computer Society.

Silva, R. and Vasconcelos, C. (2019). Realidade aumentada como apoio À aprendizagem de poliedros. Ensino da Matemática em Debate, pages 50-71. 